\title{
Fitoterapia na estratégia de Saúde da Família: uma revisão à luz do papel do enfermeiro
}

\section{Maria Izabel Cavalcanti da Silva Barros ${ }^{1}$ e Izabel Cavalcanti Barros Lamenha Pinto ${ }^{2}$}

${ }^{1}$ Centro Universitário de João Pessoa-UNIPÊ. Curso de Bacharelado em Enfermagem. Rodovia BR-230, km 22, S/№. Água Fria. João Pessoa-PB, Brasil (CEP 58053-000).

${ }^{2}$ Centro Universitário de João Pessoa - UNIPÊ. Rodovia BR-230, km 22, S/№. Água Fria. João Pessoa-PB, Brasil (CEP 58053-000). E-mail: izabellamenha@gmail.com.

Resumo. Este artigo tem como objetivo realizar um levantamento bibliográfico quanto ao papel do enfermeiro aplicando a Fitoterapia como prática integrativa complementar na estratégia de Saúde da Família. Trata-se de uma revisão integrativa realizada nas bases de dados eletrônicas Biblioteca Virtual de Saúde (BVS), que abrange a Literatura Latino-Americana e do Caribe em Ciências da Saúde (LILACS) e o Banco de Dados em Enfermagem (BDENF). Como resultado, foram encontrados nove artigos e, após aplicado os critérios de exclusão e inclusão, finalizaram quatro artigos para o presente estudo. É notório que o papel do enfermeiro aplicando a fitoterapia como prática integrativa complementar na estratégia de Saúde da Família é um assunto pouco relacionado. É possível perceber uma enorme escassez no que diz respeito ao conhecimento da fitoterapia para que seja feita a implantação na atenção primária. É necessário que sejam investidas novas políticas de formação e processos de capacitação, principalmente na Enfermagem, que possui contato direto com a população, sendo necessária também uma reorientação do modelo de atenção à saúde para que os profissionais tornem-se capacitados para atuar nesta forma de tratamento, já que a fitoterapia visa à promoção, à prevenção e à recuperação, de maneira viável e benéfica, trazendo melhorias para a população e para o próprio sistema de saúde.

Palavras-chave: Fitoterapia; Estratégia de saúde da família; Enfermagem.

Abstract. Phytotherapy in the Family Health strategy: A review of the light of the nurse's role. This article aims to make a bibliographic survey about the role of the nurse applying Phytotherapy as Complementary Integrative Practice in the Family Health Strategy. This is a integrative review of models, based on the eletronic databases of the Virtual Health Library (BVS), which covers the Latin-American and Caribbean Literature on Health Sciences (LILACS) and Nursing Databases (BDENF).
Recebido

$19 / 10 / 2021$

Aceito

$24 / 11 / 2021$

Disponível on line

$24 / 11 / 2021$

Publicado

$31 / 12 / 2021$

Acesso aberto

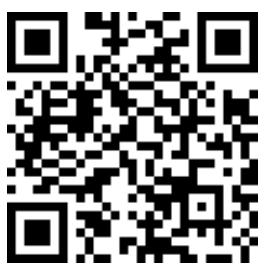

ORCID

0000-0003-3528-7307

Maria Izabel

Cavalcanti da Silva

Barros

(D) 0000-0002-7562-6310

Izabel Cavalcanti

Barros Lamenha Pinto

Rev. Bras. Gest. Amb. Sustent.

http://revista.ecogestaobrasil.net 
After the research, nine articles were found and, after applying the exclusion and inclusion criteria, four articles finished the present study. It is notorious that the role of the nurse applying phytotherapy as PIC in FHS is a subject little related. It is possible to perceive a great shortage in the knowledge of the phytotherapy so that it is made the implantation in the primary attention. It is necessary that new training policies and training processes are invested, especially in nursing, which has direct contact with the population, being also necessary a reorientation of the health care model so that professionals become qualified to act this way in the treatment, since the phytotherapy aims the promotion, prevention and recovery, in a viable and beneficial way, bringing improvements to the population and health system itself.

Keywords: Phytotherapy; Family Health Strategy; Nursing.

\section{Introdução}

O Brasil é o país com a maior biodiversidade do planeta de acordo com a organização Internacional Botanical Gardens Conservation (BGCI). Na pesquisa, chamada de Global Tree Search foram apurados nomes de espécies e a distribuição de árvores em seus respectivos países, onde foram identificadas 60.065 árvores no total, sendo 14\%, no território brasileiro (8.715), seguido da Colômbia, com 5.776, e Indonésia, com 5.142. Entre os elementos que a compõem essa biodiversidade, estão as plantas medicinais que são matérias-primas para a fabricação de fitoterápicos entre outras categorias de medicamentos (BGCI, 2017).

0 uso das plantas medicinais no tratamento de diversos problemas de saúde foi construído com base na experiência popular, sendo transmitido através de gerações, que advém de senso comum, como forma alternativa para solucionar sintomas, principalmente para o sque não tem acesso aos serviços de saúde com facilidade (Calixto, 2003).

Lopes (2010) diz que o uso das plantas medicinais com finalidade terapêutica constitui um importante recurso para a população, verifica-se um espaço onde farmacêuticos e outros profissionais da saúde, como enfermeiros, podem trabalhar, de modo a contribuir para a utilização correta de fitoterápicos, inclusive na Estratégia de Saúde da Família (ESF) e atenção básica.

Em meados da década de 1980 diversas ações e programas de fitoterapia foram implantados na rede pública de saúde, destacando que as terapias complementares, como a Política Nacional de Práticas Integrativas e Complementares (PNPIC) para o Sistema Único de Saúde (SUS), incluídas pela Portaria GM/MS no 971/2006 (Brasil, 2006), recomenda a Fitoterapia para os aspectos promocional, preventivo, curativo e de reabilitação de diversos agravos agudos e crônicos (Nunes, 2015).

Corroborando com essa questão, o SUS implantou em 2006, a PNPIC com o objetivo de estimular a utilização da Fitoterapia para a prevenção de agravos por meio de tecnologias eficazes e seguras. Em ato seguinte, em 2009, foi implantada a Política Nacional de Plantas Medicinais e Fitoterápicos, que estabelece diretrizes e linhas prioritárias para o desenvolvimento de ações em torno de objetivos comuns voltados à garantia do acesso seguro e uso racional de plantas medicinais e fitoterápicos em nosso país (Brasil, 2006; Brasil, 2009). 
Portaria MS no 886/2010, inicia, no âmbito do SUS, a Farmácia Viva, programa no contexto da Política Nacional de Assistência Farmacêutica que realiza todas as etapas desde o cultivo à dispensação de preparações magistrais e oficinas de plantas medicinais e fitoterápicas (Brasil, 2010).

Para Souza (2010) os profissionais de saúde como médicos e enfermeiros, que possuem o intuito de transformar o uso de plantas medicinais em uma prática concreta a ser aplicada na ESF, devem ter incorporado este conhecimento, na tentativa de delimitar tal prática de cuidado, inclusive baseando-se em experiências acerca das implicações éticas e legais da aplicabilidade de plantas em tratamentos de saúde.

A Enfermagem tem entre as suas atribuições promover a Educação em Saúde. Rezende e Cocco (2002) mostraram ser relevante a execução da Enfermagem neste campo, compreendendo as plantas e a fitoterapia como forma de prevenção da cultura de práticas populares, identificando carências da população no que diz respeito ao seu uso correto, já que erros de diagnóstico, identificação incorreta de espécies de plantas e uso diferente da forma tradicional podem ser perigosos, levando a superdose, ineficácia terapêutica e ao aparecimento de reações adversas (RAMs) (OPAS, 2011).

A escolha deste tema surgiu a partir da curiosidade a respeito do papel que $o$ Enfermeiro desempenha, ao aplicar a fitoterapia na Atenção Primária enquanto Políticas Integrativas Complementares, manifestando a seguinte questão norteadora: qual o papel do Enfermeiro no manejo de plantas medicinais e fitoterápicos na estratégia de saúde da família?

Este estudo objetiva realizar um levantamento bibliográfico, no período de 2010 a 2018, quanto ao papel do Enfermeiro aplicando a Fitoterapia como Prática Integrativa Complementar na Estratégia de Saúde da Família, evidenciando os principais periódicos que retratam sobre o tema e quais as contribuições para a Enfermagem.

\section{Referencial teórico}

\section{Fitoterapia}

0 uso terapêutico das plantas tem levantado o interesse da Enfermagem, tanto em estudo científicos, participação em programa de formação continuada do Governo Federal em capacitação sobre o tema, e pelo próprio Conselho Federal de Enfermagem (COFEN), por meio da Resolução COFEN no 570/2018 (COFEN, 2018), reforçando a fitoterapia como especialidade do enfermeiro por área de abrangência.

Parte da população mundial já teve alguma experiência de utilização de plantas medicinais com fins preventivos ou curativos. As plantas medicinais foram empregadas pelo homem no tratamento de enfermidades de todos os tipos, ou seja, a utilização de plantas na prevenção e/ou na cura de doenças é um hábito que sempre existiu na História da Humanidade (Calixto, 2003).

Conforme Calixto (2003), historicamente foi em finais do século XVIII, que se começou a isolar e determinar a estrutura dos constituintes ativos dos produtos de origem natural dotados de propriedades medicinais. Os recursos terapêuticos disponíveis até o século XIX eram exclusivamente oriundos de plantas medicinais e extratos vegetais, ou seja, a utilização de plantas era barata e culturalmente difundida.

No século XX, inicia-se a tendência de se isolar os princípios ativos, realizando diversos processos englobando química orgânica durante a transformação. São eles a fitoquímica (isolamento, purificação do princípio ativo), farmacológico (investigação de extratos e constituintes químicos isolados), química orgânica sintética (transformação química dos princípios ativos), química medicinal (estuda o mecanismo de ação dos princípios ativos) e por fim a preparação e formulação dos fitoterápicos, a fim de tornar o uso das plantas medicinais (fitoterápicos) seguro para forma terapêutica (Brasil, 2005; Maciel et al., 2002). 
Nunes (2015) apresenta que maior parte dos fitoterápicos e plantas medicinais utilizadas atualmente por automedicação ou por prescrição médica não possui perfil tóxico conhecido e do ponto de vista científico, muitas plantas possuem substâncias agressivas e por essa razão devem ser utilizadas, respeitando seus riscos toxicológico.

Segundo a Agência Nacional de Vigilância Sanitária (ANVISA) os fitoterápicos são medicamentos que possuem compostos químicos, os quais podem interagir com outras categorias medicamentosas. Da mesma forma, as plantas medicinais também possuem compostos químicos ativos que podem promover este tipo de interação (ANVISA, 2004).

As plantas medicinais e, por consequência, os medicamentos fitoterápicos, são constituídos de misturas complexas de substâncias bioativas, denominados compostos secundários, que podem ser responsáveis por ações polivalentes. No medicamento fitoterápico, ao contrário do medicamento sintético, não há substância ativa isolada, o que dificulta informações acerca de sua ação farmacológica e biodisponibilidade (Alexandre et al., 2008; Machado et al., 2014).

De acordo com Organização Mundial da Saúde (OMS, 1993), biodisponibilidade consiste na rapidez e amplitude da absorção de um princípio ativo de determinada forma farmacêutica, sendo determinado pela sua curva de concentração e tempo na circulação sistêmica ou pela sua excreção urinária.

É responsabilidade das autoridades regulatórias nacionais assegurar a execução das análises necessárias para garantir a qualidade e eficácia dos produtos circulando no mercado. A OMS recomenda a padronização de estudos de equivalência de medicamentos, com a finalidade de que sejam considerados intercambiáveis. Nesse sentido, foi publicado em 2018, pela ANVISA o Primeiro Suplemento do Formulário de Fitoterápicos da Farmacopeia Brasileira para uso destes fitoterápicos (Bermudez, 1994).

Uma das preocupações com relação ao uso de plantas medicinais e fitoterápicos advém do fato de que muitos usuários têm a falsa ideia de que tais produtos são isentos de efeitos tóxicos e/ou colaterais por serem "naturais". Com tal premissa, parte considerável dos usuários não revela aos prescritores sobre a utilização de produtos à base de plantas medicinais (Machado et al., 2014).

Assim, a Fitoterapia é encarada como opção na busca de soluções terapêuticas, utilizada principalmente pela população de baixa renda, já que se trata de uma alternativa eficiente, barata e culturalmente difundida (Lopes, 2010). Para Saad et al. (2016, p. 11), a Fitoterapia Moderna é exercida dentro de diferentes paradigmas e observa-se grande tendência à hibridização de conhecimentos proporcionada pela facilidade de troca de informações entre as várias culturas. A associação de conceitos comuns aos sistemas médicos tradicionais consagrados, a incorporação de novos conhecimentos terapêuticos e de métodos diagnósticos advindos das pesquisas científicas têm sido a tendência seguida. Essa abordagem possibilita uma ampliação da visão sobre o ser humano, trazendo novas formas de pensar o ajusteorgânico. 0 conhecimento científico sobre as plantas utilizadas pela população ainda é escasso. Segundo Silva e Hans (2011), as pesquisas realizadas nessa área objetivam fornecer informações adequadas para sua utilização. Qual parte da planta deve ser utilizada, como prepará-la e quais as potenciais interações podem ocorrer quando utilizadas concomitantemente ao tratamento farmacológico são alguns pontos que devem seresclarecidos.

A população em geral confunde a Fitoterapia com o uso de plantas medicinais. A ANVISA considera como medicamento fitoterápico aquele obtidos com emprego exclusivo de matérias primas ativas vegetais cuja segurança e eficácia sejam baseadas em evidências clínicas e que sejam caracterizados pela constância de sua qualidade.

São considerados produtos tradicionais fitoterápicos os obtidos com emprego exclusivo de matérias-primas ativas vegetais cuja segurança e efetividade sejam baseadas em dados de uso seguro e efetivo publicados na literatura 
técnico-científica e que sejam concebidos para serem utilizados sem a vigilância de um médico para fins de diagnóstico, de prescrição ou de monitorização (art. 2ํㅡㄴ §§

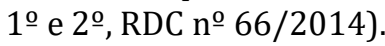

\section{Prática integrativa complementar: fitoterapia}

Observam-se iniciativas que têm como objetivo revalorizar teorias há muito esquecidas no Ocidente, em um movimento de aproximação da Ciência com os saberes tradicionais considerados não científicos. Assim em 2011, surge o reconhecimento da Organização Pan-Americana de Saúde/Organização Mundial da Saúde (OPAS/OMS), que, ciente da sua importância, define a medicina tradicional como o total de conhecimento técnico e procedimentos com base nas teorias, crenças e experiências de diferentes culturas, explicáveis ou não pela Ciência e usados para a manutenção da saúde, prevenção, diagnoss e tratamento de doenças físicas e mentais (OPAS, 2011).

A OMS estabeleceu como meta reduzir em $2 \%$ ao ano, até 2015 , a incidência de doenças crônicas. Sabe-se que, no Brasil, menos de $50 \%$ da população tem acesso às medicações essenciais para o tratamento de doenças crônicas (OPAS, 2011).

Dentre essas questões e em virtude da opção alternativa de tratamento que vem sendo cada vez mais utilizada na rede básica de saúde de diversas cidades brasileiras, o COFEN, por meio da Resolução COFEN no 570/2018, atualizou, no âmbito do Sistema COFEN/Conselhos Regionais de Enfermagem, os procedimentos para registro de títulos de pós-graduação lato e stricto sensu concedido a Enfermeiros e lista as especialidades, incluindo na Área I - Saúde Coletiva; Saúde da Criança e do adolescente; Saúde do Adulto (Saúde do Homem e Saúde da Mulher; Saúde do Idoso; Urgências e Emergências), a Fitoterapia (COFEN, 2018).

\section{Fitoterapia na atenção primária}

0 uso terapêutico de plantas medicinais tem despertado um grande interesse por profissionais da área da saúde, ao longo dos anos têm sido registrados variados procedimentos clínicos tradicionais utilizando plantas medicinais, que são assim definidas adquirir elementos que têm ação farmacológica, estas substâncias são definidas como princípios ativos (Veiga Júnior, 2005).

Entende-se por qualidade o conjunto de critérios que caracterizam a matériaprima para o uso ao qual se destina. Portanto, a qualidade da matéria-prima vegetal é a determinante inicial da qualidade do fitoterápico (Farias, 1999). Segundo este autor, somente a qualidadedas matérias-primas vegetais não garante, por si, a eficácia, a segurança e a qualidade do produto final. A eficácia é dada pela comprovação, através de ensaios farmacológicos pré-clínicos e clínicos, dos efeitos biológicos preconizados para esses recursos terapêuticos.

Em 1994, o Ministério da Saúde (MS) criou o Programa de Saúde da Família (PSF), com o propósito de reorganizar a prática da atenção à saúde, surgindo assim como uma nova maneira de trabalhar em saúde, tendo a família como centro da atenção e desenvolvendo uma nova visão da comunidade em que não se espera que o indivíduo busque por assistência, e, sim, que aja preventivamente (Sampaio et al., 2013).

O PSF tem por objetivo contribuir para a reorientação do modelo assistencial a partir da atenção básica, conforme os princípios de universalização, descentralização, integralidade e participação da comunidade, construindo uma nova maneira de atuação nas unidades básicas de saúde (Brasil, 1998). Desta forma, o mesmo busca integrar ações voltadas para osvários aspectos da saúde dos indivíduos, identificando soluções para os mais diversos problemas, representando uma concepção de saúde centrada na promoção da qualidade devida.

Na concepção do PSF, o enfermeiro atua como coordenador da equipe da saúde, criando um maior vínculo com a comunidade através de suas ações de prevenção e 
promoção à saúde, contribuindo para o aumento da qualidade da assistência prestada (Sampaio et al., 2013).

A realidade de grande parte da população brasileira, ainda hoje, é marcada pela precariedade e desigualdade no que diz respeito ao acesso aos medicamentos e tratamentos médicos necessários, sendo predominante nos usuários da atenção básica, culminando para a busca crescente em terapias alternativas a fim de melhorar a qualidade da saúde, dentre as quais destaca a fitoterapia, onde tais produtos são obtidos através das plantas medicinais, com finalidade profilática, curativa ou paliativa (Sampaio et al.,2013).

Apesar de ainda escassa, na atualidade, percebe-se o interesse de profissionais de diversas áreas relacionado à fitoterapia, mesmo não ocorrendo seu uso de modo orientado, visto que a maior parte das orientações surgem do saber popular e não do embasamento científico. Neste contexto, o enfermeiro surge como peça-chave para buscar a melhoria dos tratamentos fitoterápicos, principalmente os vinculados à ESF que possuem maior vínculo com a comunidade e maior acessibilidade ao atendimento em saúde. O papel do enfermeiro em orientar e assistir a população a respeito dos fitoterápicos continua sendo deficiente, seja por falta de conhecimento científico, deficiência no sistema acadêmico ou até por falta de interesse na área (Sampaio et al.,2013).

Em 2006, o Ministério da Saúde desenvolveu o Plano Nacional de Práticas Integrativas e Complementares (PNPIC), incorporando as práticas alternativas em saúde na perspectiva da prevenção e promoção da saúde, com ênfase na atenção básica, considerando as práticas alternativas, como a fitoterapia, como recursos que estimulam os mecanismos naturais de prevenção e recuperação da saúde, através de tecnologias seguras e eficazes, com ênfase na escuta acolhedora, no desenvolvimento do vínculo terapêutico, integrando o ser humano ao meio ambiente e a sociedade (Sampaio et al.,2013).

A implantação e o fortalecimento da Fitoterapia na rede de saúde é uma questão de cidadania e dá-se na medida em que favorece a participação da população no entendimento da intervenção médica no seu organismo como também no sentido de fazer com que ela saia do seu papel de passividade e seja um agente ativo no cuidado a saúde (Bastos e Lopes, 2010).

0 fato do enfermeiro ser a peça-chave da ESF, esse contato possibilita, conforme Brasileiro et al. (2008), conhecer a cultura e o saber popular de determinada comunidade, e, assim, empregá-los no processo saúde doença damesma.

Segundo Arnous et al. (2005), a população confia no tratamento com os medicamentos fitoterápicos por ser natural, de baixo custo e de fácil acesso. Ao contrário do que ocorre com medicamentos alopáticos, a fitoterapia é um método de cura e prevenção mais acessível para a população, com políticas públicas voltadas a essa realidade e tendo o enfermeiro papel na atenção básica, para orientação da população carente, estabelece-se uma relação significativa, que pode ser explorada na ESF. A participação do enfermeiro na orientação da população é de grande valor, o uso incorreto pode desencadear RAMs, além dos riscos de interações com outros medicamentos ou alimentos, ou ainda relacionados a características do paciente.

\section{Metodologia}

Trata-se de uma revisão integrativa, constituída por todos os artigos selecionados que abordam o papel do enfermeiro aplicando a fitoterapia como Prática Integrativa Complementar na Estratégia de Saúde da Família, publicados em português, indexados nas bases de dados eletrônicas da Biblioteca Virtual de Saúde (BVS), que abrange a Literatura Latino-Americana e do Caribe em Ciências da Saúde (LILACS), e o Banco de Dados em Enfermagem (BDENF), publicados no período de 2010 a 2018, tendo como descritores 
"Fitoterapia", "Estratégia de Saúde da Família" e "Enfermagem", combinados pelo conector booleano "AND".

Quanto aos critérios de exclusão, foram artigos em duplicidade, artigos incompletos, fora do período de publicação pré-estabelecido, tese, dissertações e monografias.

A coleta de dados foi realizada entre os meses de agosto e outubro de 2018, seguindo os passos metodológicos da revisão integrativa da literatura, composta por seis fases, sendo a primeira fase correspondente a elaboração da questão, a segunda ao estabelecimento da estratégia de busca na literatura, a terceira sendo a seleção de estudos com base nos critérios de inclusão, a quarta a leitura crítica/avaliação/categorização dos conteúdos, a quinta concernente a análise e a sexta a interpretação dos resultados (Souza et al., 2010).

Por fim, foi construído um instrumento de coleta de dados contendo a identificação do artigo incluindo título, autores, método, base de dados, ano de publicação, objetivo do estudo e principais resultados, discussões relevantes e principais achados nas conclusões.

Os dados coletados foram apresentados por representações em tabelas e os achados foram analisados qualitativamente, para responder aos objetivos propostos por esta pesquisa.

\section{Resultados e discussão}

Foram encontrados nove artigos relacionados, onde oito estão na língua portuguesa, dentre eles dois são de duplicidade, um é uma tese e um está fora do período estabelecido, sendo estes excluídos a partir dos critérios de exclusão, finalizando um total de quatro $(\mathrm{n}=4)$ artigos para análise. Destes quatro artigos, dois (50\%) encontram-se no Banco de dados em enfermagem (BDENF) e dois (50\%) na Literatura Latino-Americana e do Caribe em Ciências da Saúde(LILACS) (Figura 1).

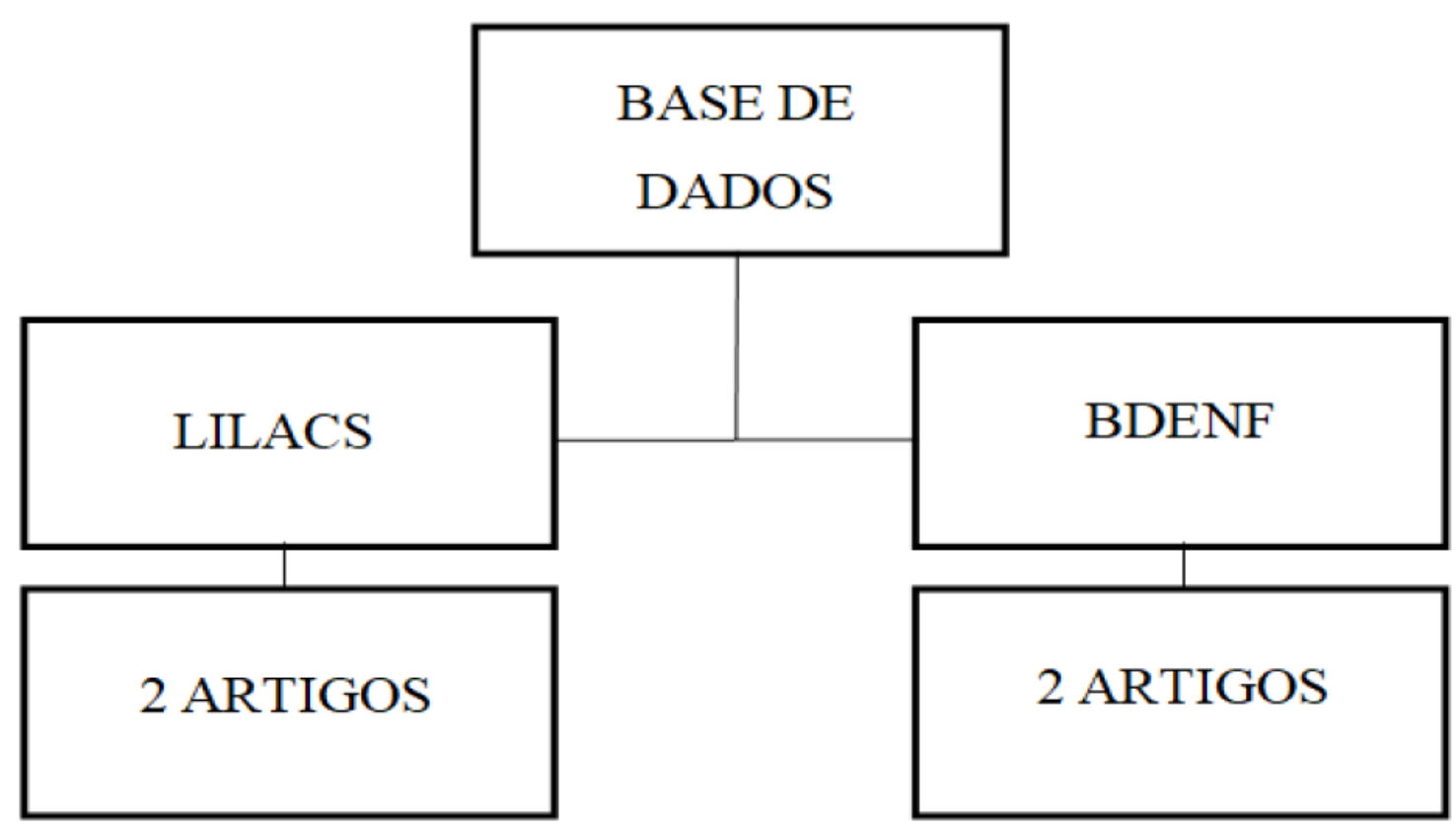

Figura 1. Descrição das bases de dados. 
A Tabela 1 apresenta a distribuição dos artigos por base e dados eletrônicas, contendo título, ano, autores, objetivos e abordagem.

Tabela 1. Descrição dos artigos quanto ao título, autores, ano, objetivos, abordagem e base de dados.

\begin{tabular}{|c|c|c|c|c|}
\hline Artigo & 1 & 2 & 3 & 4 \\
\hline Título & $\begin{array}{c}\text { Dificuldades de } \\
\text { profissionais de } \\
\text { saúde frente ao uso } \\
\text { de plantas } \\
\text { medicinais e } \\
\text { fitoterápicos. }\end{array}$ & $\begin{array}{c}\text { Percepção dos } \\
\text { enfermeiros da } \\
\text { estratégia saúde da } \\
\text { família sobre o uso } \\
\text { da fitoterapia. }\end{array}$ & $\begin{array}{l}\text { Terapêutica com plantas } \\
\text { medicinais: percepção } \\
\text { de profissionais da ESF } \\
\text { de um município em PE. }\end{array}$ & $\begin{array}{l}\text { A Fitoterapia na } \\
\text { Rede Básica de } \\
\text { Saúde: o Olhar } \\
\text { daEnfermagem }\end{array}$ \\
\hline Autores & Varela e Azevedo & Sampaio et al. & Menezes et al. & Bastos e Lopes \\
\hline Ano & 2013 & 2013 & 2012 & 2010 \\
\hline Objetivo & \begin{tabular}{|c} 
Identificar as \\
dificuldades \\
encontradas por \\
médicos e \\
enfermeiros na \\
aplicabilidade de \\
plantas medicinais \\
e fitoterápicos na \\
(ESF) de Caicó/RN.
\end{tabular} & $\begin{array}{c}\text { Conhecer a } \\
\text { percepção dos } \\
\text { enfermeiros sobre o } \\
\text { uso dafitoterapia na } \\
\text { Estratégia Saúde da } \\
\text { Família. }\end{array}$ & \begin{tabular}{|} 
Verificar a percepção de \\
médicos, cirurgiões- \\
dentistas e enfermeiros \\
da ESFno município de \\
Caruaru, PE, Brasil, \\
sobre a importância, \\
utilização e indicações \\
de plantasmedicinais
\end{tabular} & \begin{tabular}{|c|} 
Avaliar o \\
conhecimento que \\
oprofissional de \\
Enfermagem tem \\
sobre Fitoterapia e \\
as dificuldades \\
encontradas para \\
implementação \\
dessa terapêutica \\
nas USF. \\
\end{tabular} \\
\hline Abordagem & Qualitativa & Qualitativa & Quantitativo (transversal) & Quantiqualitativa \\
\hline Base dados & BDENF & BDENF & LILACS & LILACS \\
\hline
\end{tabular}

Após a análise dos artigos, surgiram duas categorias, a percepção da fitoterapia pelos profissionais de saúde e a dificuldade dos profissionais de saúde frente ao uso da fitoterapia.

\section{A percepção da fitoterapia pelos profissionais de saúde}

A terapia com plantas medicinais era conhecida e praticada pelas antigas civilizações, sendo a fitoterapia uma forma de tratamento milenar, simples e natural, que busca a cura e previne doenças, através das preparações vegetais a baseada no mesmo princípio do medicamento alopático, se tornando, com o decorrer do tempo, uma terapia complementar, visando à prevenção, ao tratamento ou à cura (Bastos e Lopes, 2010).

Na perspectiva do PSF, o enfermeiro possui papel destacado como coordenador da equipe de saúde, possuindo um maior vínculo com a comunidade, já que consegue por meio de suas ações prevenir e promover a saúde, contribuindo para uma assistência qualificada, onde, nesse contato, é possível conhecer a cultura e o saber popular de determinada população, conseguindo empregá-los no processo saúde doença, onde é ressaltado o interesse da enfermagem nesta área (Sampaio et al., 2013; COFEN, 2018).

Segundo Sampaio et al. (2013), ainda há deficiência à respeito sobre o conhecimento da fitoterapia enquanto prática complementar, bem como as políticas que a respaldam, tornando-se necessário maior orientação e incentivo na busca do conhecimento para que sua utilização seja realizada de forma segura e correta, beneficiando sistema e população, corroborando Bastos e Lopes (2010) que diz que o conhecimento formal da enfermagem relacionado à fitoterapia ainda é insuficiente, 
destacando a necessidade de novas capacitações para que os profissionais possam aplicar a Fitoterapia como Prática Integrativa Complementar.

De acordo com Menezes et al. (2012), torna-se importante, na visão dos profissionais de saúde a implantação de medicamentos alternativos na atenção básica, visando seu baixo custo, eficácia e fácil acesso da população.

\section{A dificuldade dos profissionais de saúde frente ao uso dafitoterapia}

Ao olhar dos profissionais de saúde, há uma certa resistência da população ao uso de fitoterápicos, seja pela supervalorização dos medicamentos alopáticos e novas tecnologias e/ou pela descrença cultural concedido a estes produtos no decorrer do tempo, onde tal percepção acaba desprezando as Práticas Integrativas como forma de tratamento, já que existe um preconceito que mostra como argumento o abandono da medicina alopática e conhecimento cientifico para a medicina alternativa e complementar, taxadas como irracionaise desnecessárias. A partir do momento que a população começa a usufruir de novos recursos, esquece e despreza o saber relacionado às demais terapias existentes (Varela et al., 2013).

Tratando deste tema, com Varela et al. (2013), relata que outra dificuldade encontrada frente ao uso da Fitoterapia foi o conhecimento dos profissionais sobre esta temática. Muitos atribuem o fato à ausência de conteúdo durante as graduações, já que as grades curriculares que ofertam a disciplina de Fitoterapia são mínimas. Outros motivos encontrados para tal déficit foram a falta de embasamento cientifico, discriminação, falta de tempo e desconhecimento acerca do assunto, tornando os profissionais incapacitados para exercer funções de prevenção e promoção de saúde com a fitoterapia, como também a ausência dos insumos, configurando- se num problema de ordem econômica para os usuários obteremacesso.

Ainda é afirmado que o saber popular apresenta algumas concepções equivocadas em torno da fitoterapia, acabando por dificultar a aplicabilidade dessa terapêutica na ESF. Existe uma ideia de que os produtos naturais são inofensivos à saúde, de acordo com a maioria dos usuários, o que pode desencadear saberes e práticas que podem fugir da finalidade terapêutica (Varela et al., 2013).

\section{Considerações finais}

Nesta revisão foi exequível analisar o papel do enfermeiro aplicando a Fitoterapia como Prática Integrativa Complementar na Estratégia de Saúde da Família que, mesmo com poucos artigos relacionados ao tema, foi possível observar que apesar da fitoterapia ser um tratamento complementar de suma importância para a população, o conhecimento sobre a mesma ainda é escasso, trazendo dificuldades para sua propagação no âmbito da Estratégia de Saúde da Família, inviabilizando o profissional de enfermagem aplicá-la como prática integrativa. Na atualidade, o processo do cuidar está voltado em sua grande maioria, para a medicina tradicional, ou seja, o uso de medicamentos alopáticos, fazendo com que os profissionais de saúde fiquem sempre na mesma forma de tratamento, não se interessando pela medicina alternativa, que está sendo deixada de lado, por falta de embasamento científico, formação deficiente e preconceito com a cultura popular, emgeral.

A fim de reverter essa sitauação faz-se necessário que sejam investidas novas políticas de formação e processos de capacitação, onde as Políticas Integrativas Complementares façam parte da formação acadêmica dos profissionais, principalmente na Enfermagem, para que se conheça as propriedades das plantas medicinais/fitoterápicos para que o enfermeiro possa realizar intervenções educativas, instruindo a população ao uso correto buscando as finalidades terapêuticas. 
Faz-se necessário também uma reorientação do modelo de atenção à saúde para que os profissionais se tornem capacitados para atuar nesta forma de tratamento, já que a Fitoterapia visa à promoção, à prevenção e à recuperação, de maneira viável e benéfica, trazendo melhorias para a população e o próprio sistema de saúde.

\section{Conflito de interesses}

As autoras declaram não haver conflito de interesses.

\section{Referências}

Alexandre, R. F.; Bagatini, F.; Simões, C. M. O. Interações entre fármacos e medicamentos fitoterápicos à base de ginkgo ou ginseng. Revista Brasileira de Farmacognosia, v, 18, n. 1, p. 117-126, 2008. https://doi.org/10.1590/S0102-695X2008000100021

Arnous, A. H.; Santos, A. S.; Beinner, R. P. C. Plantas medicinais de uso caseiro: conhecimento popular e interesse por cultivo comunitário. Revista Espaço para a Saúde, v. 6, n. 2, p. 1-6, 2005.

Bastos, R. A. A.; Lopes, A. M. C. A Fitoterapia na Rede Básica de Saúde: o olhar da Enfermagem. Revista Brasileira de Ciências da Saúde, v. 14, n. 2, p. 21-28, 2010.

BGCI - Banco de dados online da Global Tree Search. Jardins Botânicos Conservação Internacional. Richmond, Reino Unido: BGCI, 2017. Disponível em: <http://www.bgci.org/ globaltree_search.php>. Acesso em: 17 ago. 2021.

Brasil. Resolução ANVISA no 66, de 26 novembro de 2014. Altera o Anexo IV da Resolução da Diretoria Colegiada - RDC no 26, de 13 de maio de 2014, que dispõe sobre o registro de medicamentos fitoterápicos e o registro e a notificação de produtos tradicionais fitoterápicos. Disponível em: <https://bvsms.saude.gov.br/bvs/saudelegis/ anvisa/2014/rdc0066_26_11_2014.pdf>. Acesso em: 17 ago. 2021.

Brasil. Ministério da Saúde. Política Nacional de Medicina Natural e Práticas Complementares - PMNPC. Brasília: MS, 2005. (Resumo executivo). Disponível em: $<$ http://bvsms.saude.gov.br/bvs/publicacoes/ResumoExecutivoMedNatPratCompl14020 52.pdf>. Acesso em: 21 mar. 2021.

Brasil. Ministério da Saúde. Política Nacional de Práticas Integrativas e Complementares no SUS - PNPIC-SUS. Brasília: MS, 2006. Disponível em: $<$ http://bvsms.saude.gov.br/bvs/publicacoes/politica_nacional_praticas_integrativas_com plementares_2ed.pdf>. Acesso em: 22 mar. 2021.

Brasil. Ministério da Saúde. RENISUS - Relação Nacional de Plantas Medicinais de Interesse ao SUS. 2009. Disponível em: <https://portalarquivos2.saude.gov.br/images/pdf/ 2014/maio/07/renisus.pdf>. Acesso em: 21 mar. 2018.

Brasil. RDC no 48, de 16 março de 2004. Disponível em: <https://www.arca.fiocruz.br/ bitstream/icict/19131/2/11.pdf>. Acesso em: 21 mar. 2018.

Brasileiro, B. G.; Pizziolo, V. R.; Matos, D. S.; Germano, A. M.; Jamal, C. M. Plantas medicinais utilizadas pela população atendida no "Programa de Saúde da Família", Governador Valadares, MG, Brasil. Revista Brasileira de Ciências Farmacológicas, v. 44, n. 4, p. 112-117, 2008. https://doi.org/10.1590/S1516-93322008000400009

Calixto, J. B. Biodiversidade como fonte de medicamentos. Ciência e Cultura, v. 55, p. 37-39, 2003. 
COFEN - Conselho Federal de Enfermagem. Resolução COFEN no 581/2018. Disponível em: <http://www.cofen.gov.br/resolucao-cofen-no-0570-2018_61172.html>. Acesso em: 26 fev. 2021.

Cunha, A. P. Aspectos históricos sobre plantas medicinais, seus constituintes activos e fitoterapia. In: Cunha, A. P.; Roque, O. R.; Silva, A. P. Plantas e produtos vegetais em fitoterapia. Lisboa: Fundação Calouste Gulbenkian, 2003.

Farias, M. R. Avaliação da qualidade de matérias-primas vegetais. In: Simões, C. M. O.; Schenkel, E. P.; Gosmann, G.; Mello, J. C. P.; Mentz, L. A.; Petrovick, P. R. (Orgs.). Farmacognosia: da planta ao medicamento. Porto Alegre: Ed. UFRGS; Florianópolis: Ed. da UFSC, 1999. p. 197-220.

Machado, H. L.; Moura, V. L.; Gouveia, N. M.; Costal, G. A.; Espindola, F. S.; Botelho, F. V. Pesquisa e atividades de extensão em fitoterapia desenvolvidas pela Rede FitoCerrado: uso racional de plantas medicinais e fitoterápicos por idosos em Uberlândia-MG. Revista Brasileira de Plantas Medicinais, v. 16, n. 3, p. 527-533, 2014. https://doi.org/10.1590/ 1983-084X/13_072

Nunes, M. G. S.; Bernardino, A.; Martins, R. D. Uso de plantas medicinais por pessoas com hipertensão. Revista Rene, v. 16, n. 6, p. 775-781, 2015. https://doi.org/10.15253/21756783.2015000600002

OPAS - Organização Pan-Americana de Saúde. Boas práticas de farmacovigilância para as Américas. Washington: OPAS, 2011. Disponível em: <http://apps.who.int/medicinedocs/documents/s18625pt/s18625pt.pdf>. Acesso em: 21 mar. 2021.

Rezende, H. A.; Cocco, M. I. M. A utilização de fitoterapia no cotidiano de uma população rural. Revista Escola de Enfermagem USP, v. 36, n. 3, p. 282-288, 2002. https://doi.org/10.1590/S0080-62342002000300011

Saad, G. A.; Léda, P. H. O.; Sá, I. M.; Seixlack, A. C. C. Fitoterapia contemporânea: tradição e ciência na prática clínica. 2. ed. Rio de Janeiro: Guanabara Koogan, 2016.

Sampaio, L. A.; Oliveira, D. R.; Kerntopf, M. R.; Brito Júnior, F. E.; Menezes, I. R. A. Percepção dos enfermeiros na estratégia saúde da família sobre o uso da fitoterapia. Revista Mineira de Enfermagem, v. 17, n. 1, p. 76-84, 2013. https://doi.org/10.5935/14152762.20130007

Santos, R. L.; Guimaraes, G. P.; Nobre, M. S. C.; Portela, A. S. Análise sobre a fitoterapia como prática integrativa no Sistema Único de Saúde. Revista Brasileira de Plantas Medicinais, v. 13, n. 4, p. 486-91, 2011. https://doi.org/10.1590/S1516-05722011000400014

Souza, M. T.; Silva, M. T.; Cravalho, R. Revisão integrativa: o que é e como fazer. Einstein, v. 8, n. 1, p. 102-106, 2010. https://doi.org/10.1590/S1679-45082010RW1134

Veiga Júnior, V. F.; Pinto, A. C.; Maciel, M. A. M. Plantas medicinais: cura segura? Química Nova, v. 28, n. 3, p. 519-528, 2005. https://doi.org/10.1590/S0100-40422005000300026

Informação da Licença: Este é um artigo Open Access distribuído sob os termos da Licença Creative Commons Attribution, que permite uso irrestrito, distribuição e reprodução em qualquer meio, desde que a obra original seja devidamente citada. 